\title{
Mortality and Length of Stay in Patients with Bloodstream Infections Due to Drug-Susceptible Versus Drug-Resistant Gram-Negative Bacteria
} \author{
Mahmoud Barbarawi ${ }^{2}$ \\ ${ }^{1}$ Department of Medicine, School of Medicine, University of Jordan, Amman, Jordan \\ ${ }^{2}$ Department of Internal Medicine, Al Takhassusi Hospital, Amman, Jordan \\ ${ }^{3}$ Department of Pharmacy, Al Khalidi Hospital, Amman, Jordan \\ ${ }^{4}$ Department of Medicine, Jordan Hospital, Amman, Jordan \\ ${ }^{5}$ Department of Pharmacy, Jordan Hospital, Amman, Jordan \\ ${ }^{6}$ Department of Intensive Care Unit, Al Khalidi Hospital, Amman, Jordan
}

Jamal Wadi Al Ramahi ${ }^{1, ~ *, ~ A h m a d ~ A l-A b d o u h ², ~ N o u r ~ H a s a n ~}{ }^{3}$, Ghayda Haddad $^{3}$, Mohammad Al Baba ${ }^{2}$, Ahmad Al Aaraj ${ }^{2}$, Mahmoud Fakhri ${ }^{4}$, Lamya Abu Shanab ${ }^{5}$, Belal Harara ${ }^{6}$,

\section{Email address:}

jamalwadimd@yahoo.com (J. W. Al Ramahi), ahmad_alabdouh@yahoo.com (A. Al-Abdouh), Nr.hasan@yahoo.com (N. Hasan), ghayda.hadda@yahoo.com (G. Haddad), albaba_88@hotmail.com (M. Al Baba), ahmadalaaraj@yahoo.com (A. Al Aaraj), mahmoudfakhri88@gmail.com (M. Fakhri),lamyaabushanab@hotmail.com (L. A. Shanab), dr.harara@gmail.com (B. Harara), mahmoud.albarbarawi@gmail.com (M. Barbarwi)

${ }^{*}$ Corresponding author

\section{To cite this article:}

Jamal Wadi Al Ramahi, Ahmad Al-Abdouh, Nour Hasan, Ghayda Haddad, Mohammad Al Baba, Ahmad Al Aaraj, Mahmoud Fakhri, Lamya Abu Shanab, Belal Harara, Mahmoud Barbarawi. Mortality and Length of Stay in Patients with Bloodstream Infections Due to DrugSusceptible Versus Drug-Resistant Gram-Negative Bacteria. International Journal of Infectious Diseases and Therapy. Vol. 4, No. 3, 2019, pp. 33-39. doi: 10.11648/j.ijidt.20190403.11

Received: July 26, 2019; Accepted: August 20, 2019; Published: September 6, 2019

\begin{abstract}
A prospective patient surveillance and analysis in three urban hospitals with the objective of comparing the mortality rates among patients with antimicrobials-sensitive versus -resistant gram-negative bacterial bloodstream infections. The analysis focused on the rates of in-hospital and 28-days mortality. There were 189 patients with BSI, drug-susceptible gram-negative bacteria (DSGNB) 40.7\%, multi-drug resistant bacteria (MDRGNB) 42.3\% and extensive-drug resistant bacteria (XDRGNB) $16.9 \%$. The mean age, gender, SOFA score on the initial evaluation, APACHE II score, comorbidities, identified bacterial species, and BSI-associated diagnoses were not statistically different except for VAP $(\mathrm{P}=0.000)$ in the XDRGNB infected patients. In-hospital and 28-days mortalities were significantly higher in the XDRGNB-BSI group $(\mathrm{P}=$ $0.000)$, and ICU length of stay $(P=0.000)$. XDRGNB-BSI was significantly higher in inappropriate and delayed treated patients $(\mathrm{P}<0.05)$. Logistic regression analysis demonstrated no significant interaction for the 28 days mortality neither with the admission diagnoses, the antimicrobial class (except aminoglycosides), the comorbidities (except for solid tumors) ( $\mathrm{P}>$ 0.05 , Nagelkerke $\mathrm{R}^{2}<0.4$ ). In conclusion, BSI due to multiple class antimicrobial resistance has higher mortality and ICU length of stay.
\end{abstract}

Keywords: Multidrug-resistant Bacteria, Extensive Drug-resistant Bacteria, Bloodstream Infection, Appropriate Therapy, ICU Length of Stay

\section{Introduction}

Rug (Antimicrobials)-resistant gram-negative bacterial infections (DRGNB), especially pathogens included in the mnemonic "ESKAPE" (Enterococcus faecium, Staphylococcus aureus, Klebsiella pneumonia, Acinetobacter baumannii, 
Pseudomonas aeruginosa, and Enterobacter species) cause significant morbidity and mortality when are associated with bloodstream infection (BSI), they compromise the usefulness of the currently available antimicrobial agents, with an escalating need for complicated treatment regimens. [1]. Moreover, drugresistant gram-negative bacteria (DRGNB) with high levels resistance to multiple antimicrobials classes like multidrugresistant and extensive drug resistance (MDR and XDR)-gramnegative bacteria need complex antimicrobials regimens and clinically may do poorer $[2,3]$. Some earlier studies showed no difference in mortality between DRGNB and DSGNB in critically ill patients with nosocomial BSI [4], those patients were critically sick, had nosocomial infections and with severe morbidities, e.g. immunosuppression was associated with high mortality $(\mathrm{OR}=8.06)$ and illness severity with high bacteremia (Pitt) score $\geq 4$ (OR =18.53) [5]. In contrast, in solid organ transplant patients with DRGNB-BSI the overall mortality rate on day 30 was higher $(35.2 \%$ vs. $14.4 \% ; \mathrm{P}=0.001)$, but inappropriate empirical antibiotic therapy was identified at a higher rate among patients with DRGNB-BSI (41\% vs. $21.6 \%$; $\mathrm{P}=0.01)$, this excess mortality was not clear whether it was due to DRGNB-BSI per se or due to an added delay and inappropriate antimicrobial therapy [6]. Our objective of the current study is to compare the outcome for patients who develop DSGNB-BSI versus DRGNB-BSI whether multidrugresistant (MDRGNB-BSI) or XDRGNB-BSI, when treated with appropriate antimicrobial agents, adjusted for similar severity scores (APACHE 2 and SOFA) and all received appropriate antimicrobial therapy. [7].

\section{Materials and Methods}

\subsection{Study Setup and Location}

Prospective patient surveillance and data collection in three urban hospitals: Al Khalidi Hospital and Medical Center, the Specialty Hospital, and Jordan Hospital and Medical Center, the last two are Jordan Medical Council- and the Arab Board of medical Specialties-accredited residency teaching hospitals, all located in Amman, Jordan. The three hospitals encompass around 650 beds with 53 ICU beds, they provide primary care and referral services for Amman residents, as well as referral from other Arab Countries. The study was between January 2017 to February 2019. No consent was obtained due to the nature of the study, no suggestions or changes were made during patients care.

\subsection{Data Recruitment}

Data was collected with the cooperation among Internists, ICU specialists, Medical residents, clinical pharmacists, and microbiologists. The working teams prospectively evaluated the appropriateness of the treatment regimens for the patients; appropriateness of the prescribed antimicrobial agent (s) for the clinical management of patients, appropriateness of dose and frequency. Information was obtained by following patients as they are admitted through the emergency room, blood cultures from hospitals' microbiology laboratories, admission office, morning reports, and infection control offices. Twenty-eight days after discharge, patients were followed up by phone calls. Patients were included if they were newly admitted with the diagnosis of BSI or develop BSI during their hospital stay, and were diagnosed or suspected to have pneumonia with bacteremia, bacteremic pneumonia, abdominal infection with bacteremia, bacteremic abdominal infection, urinary infection with bacteremia, bacteremic urinary tract infections, SSTI (skin and soft tissue infection) with bacteremia, bacteremic SSTI, SSSI (skin and skin structure infection) with bacteremia, bacteremic SSSI and CNS infection with bacteremia, and were $\geq 18$ years old. Patients were excluded if pregnant, were treated with an antimicrobial outside its labeled indication, e.g. tigecycline and ertapenem (both are not labeled for patients with BSI), no bacterial growth available and imminent mortality. The study was conducted in full confidentiality, treating teams were not informed of the study conduct. Study approval from the internal review boards of the participating hospitals was obtained (Data are available on request as excel and SPSS formats on request). Participating members were urged to review the "The Strengthening the Reporting of Observational Studies in Epidemiology (STROBE) Statement: Guidelines for Reporting Observational Studies" found on https://www.strobe-statement.org/index.php?id=strobe-home.

\subsection{Definitions}

The clinical outcomes for patients were defined as the following: mortality (death), Improved: subsidence of parameters (SIRS) that diagnosed BSI, and patients did not have a sequalae like an organ damage or failure upon hospital discharge. Appropriate antimicrobial therapy: the antimicrobial agent used for treatment was covering the causative agent of BSI. Inappropriate antimicrobial therapy is defined as either the antimicrobial agent that has been used for the treatment in patients with BSI was not covering the isolated bacteria at the time of microbiological diagnosis, or delay in starting the appropriate antimicrobial agent [8-10]. Sepsis and severe sepsis syndrome definitions used for this study is according to what was described and reported elsewhere [11].

Definition for DSGNB, MDRGNB, DRGNB, and Pandrug-resistant GNB (PDRGNB): MDRGNB defines as resistant to three or more classes of antimicrobials. XDRGNB defined as extensively drug-resistant (i.e. resistant to all but one or two classes). PDRGNB defined as resistant to all available classes. DSGNB: The isolate susceptibility is not included in MDR or XDR, and susceptible to most tested agents [7]. The outcome sought in this study if there were differences in in-hospital mortality and 28 days mortality, ICU length of stay and hospital length of stay among patients with DSGNB-BSI, MDRGNB-BSI or DRGNB-BSI, no PDRGNB was isolated in our current patients.

\subsection{Statistical Analysis}

Continuous variables were calculated as mean \pm SD. Multiple 
means comparison were analyzed by ANOVA and post hoc analysis by Tukey HSD assuming equal variances. Fischer exact test and Chi-square test $\left(\mathrm{X}^{2}\right)$ was used to analyze the proportions of differences among the three tested resistance patterns. P-value was considered significant for $<0.05$. Multicollinearity was assessed among CRE, ESBL-producing GNB, DSGNB, MDRGNB, and XDRGNB: the Tolerance was adequate for all and ranged $0.773-0.850$ with low Variance Inflation Factor (VIF) 1.294 - 1.314, sensing no multicollinearity and outcomes can be analyzed for the three resistance patterns without redundancy. Data processing was by SPSS (Statistical Package for Social Sciences, version 22. IBM Corporation). Calculations of APACHE 2 score was by a web paste application found on http://reference.medscape.com/calculator/apache-ii-scoringsystem. And SOFA Score calculation found on http://www.mdcalc.com/sequential-organ-failure-assessmentsofa-score/.

\section{Results}

There are 189 patients with BSI distributed as DSGNB 77
(40.7\%), MDRGNB 80 (42.3\%) and XDRGNB 32 (16.9\%). Among the three resistance patterns, the mean ages $(\mathrm{P}=$ 0.332) and gender distribution $(P=0.066)$ were not statistically different. On the initial evaluation patients were risk stratified by SOFA scores to assess the initial likelihood of mortality and statistically were not different $(\mathrm{P}=0.152)$, neither their health evaluation status measured by the APACHE 2 score $(\mathrm{P}>0.05)$ for all subcategories. The BSIassociated admission diagnoses were not different $(\mathrm{P} \geq$ $0.138)$ except for more VAP patients $(P=0.000)$ in the XDRGNB-BSI patients. Comorbidities including diabetes mellitus, immunosuppressive treatments and states, hematological and solid malignancy, kidney transplants, abdominal and other surgeries, and other chronic medical diseases were not different $(\mathrm{P}>0.05)$ among the three resistant patterns. The isolated Enterobacteriaceae, Acinetobacter and Pseudomonas species $(\mathrm{P}=0.572)$ (Figure 1), the distribution of ESBL-producing GNB, CRE, lactose fermenters and lactose non-fermenters among the three resistance patterns (DSGNB, MDRGNB, and XDRGNB) were not significantly different $\mathrm{P}=1.0$ ), (table 1 ).

Table 1. Demography and characteristics of patients diagnosed as Gram-negative Bacterial Blood Stream Infection with drug-susceptible, multidrug resistant and extensive drug resistant bacteria.

\begin{tabular}{|c|c|c|c|c|}
\hline Characteristic & $\begin{array}{l}\text { DSGNB-BSI } \\
\mathbf{N}\end{array}$ & $\begin{array}{l}\text { MDRGNB-BSI } \\
\mathbf{N}\end{array}$ & $\begin{array}{l}\text { XDRGNB-BSI } \\
\mathbf{N}\end{array}$ & P-Value** \\
\hline Total number of patients with GNB-BSI (\%) & $77(40.7)$ & $80(42.3)$ & $32(16.9)$ & --- \\
\hline $\operatorname{Age}$ Mean $( \pm$ SD) & $65(19)$ & $64(18)$ & $65(14)$ & $0.332 *$ \\
\hline \multicolumn{5}{|l|}{ Gender } \\
\hline Males & 37 & 41 & 23 & \multirow[t]{2}{*}{0.066} \\
\hline Females & 40 & 39 & 9 & \\
\hline \multicolumn{5}{|l|}{ APACHE II Score } \\
\hline$(11-20) 2$ & 27 & 20 & 7 & \multirow{4}{*}{$>0.05$} \\
\hline$(21-30) 3$ & 17 & 11 & 9 & \\
\hline$(31-40) 4$ & 4 & 9 & 7 & \\
\hline$(>40) 5$ & 1 & 1 & 2 & \\
\hline \multicolumn{5}{|l|}{ Initial SOFA Score } \\
\hline$(<5) 1$ & 14 & 19 & 3 & \multirow{3}{*}{0.152} \\
\hline$(5-9) 2$ & 44 & 42 & 14 & \\
\hline$(>14) 4$ & 2 & 2 & 2 & \\
\hline \multicolumn{4}{|l|}{ Admission Diagnoses with BSI } & \multirow{2}{*}{0.648} \\
\hline Primary BSI & 24 & 20 & 10 & \\
\hline Ventilator-Associated Pneumonia & 2 & 4 & 9 & 0.000 \\
\hline Community-Associated Pneumonia & 14 & 11 & 5 & 0.749 \\
\hline Urinary Tract Infection $\$$ & 26 & 33 & 8 & 0.247 \\
\hline Surgical Site Infection & 2 & 3 & 0 & 0.535 \\
\hline Skin and soft tissues infections & 6 & 6 & 2 & 0.961 \\
\hline Intra-Abdominal Infection & 6 & 9 & 0 & 0.138 \\
\hline Other Diagnoses & 1 & 2 & 2 & 0.339 \\
\hline \multicolumn{4}{|l|}{ Co-Morbidities } & \multirow{2}{*}{0.783} \\
\hline Diabetes mellitus & 35 & 32 & 14 & \\
\hline Immunosuppressive treatment & 9 & 12 & 4 & 0.822 \\
\hline Immunosuppressive states & 6 & 10 & 5 & 0.433 \\
\hline Hematological malignancy & 3 & 6 & 5 & 0.104 \\
\hline Other surgeries & 3 & 9 & 5 & 0.097 \\
\hline Chronic Skin Diseases & 6 & 4 & 1 & 0.089 \\
\hline Chronic Kidney Disease & 0 & 3 & 41 & 0.115 \\
\hline Chronic Liver Disease & 0 & 1 & 11 & 0.695 \\
\hline
\end{tabular}




\begin{tabular}{|c|c|c|c|c|}
\hline Characteristic & $\begin{array}{l}\text { DSGNB-BSI } \\
\mathbf{N}\end{array}$ & $\begin{array}{l}\text { MDRGNB-BSI } \\
\mathbf{N}\end{array}$ & $\begin{array}{l}\text { XDRGNB-BSI } \\
\mathbf{N}\end{array}$ & P-Value** \\
\hline Other conditions & 31 & 46 & 17 & 0.089 \\
\hline Microbiological Diagnosis & & & & 0.572 \\
\hline E. coli & 37 & 47 & 3 & \\
\hline Klebsiella pneumoniae & 16 & 18 & 8 & \\
\hline Enterobacter spp. & 7 & 4 & 1 & \\
\hline Acinetobacter spp. & 3 & 4 & 18 & \\
\hline Pseudomonas aeruginosa and spp. & 7 & 3 & 1 & \\
\hline Other GNB ${ }^{\#}$ & 7 & 4 & 1 & \\
\hline Other Resistance patterns & & & & 1.0 \\
\hline ESBL-producing GNB & 25 & 53 & 6 & \\
\hline CRE & 0 & 0 & 5 & \\
\hline Lactose fermenter & 60 & 69 & 12 & \\
\hline Lactose non-fermenter & 10 & 7 & 19 & \\
\hline
\end{tabular}

\#Other GNB: Citrobacter freudii, Enterobacter spp., Klebsiella spp., Morganella, Proteus spp., and Serratia spp.

BSI: blood stream infection. DSGNB: drug susceptible gram-negative bacteria. MDR: multidrug resistant. XDR: extensive drug resistant. ESBL-GNB: extended spectrum beta-lactamases producing gram-negative bacilli. CRE: Carbapenem-resistant Enterobacteriaceae.

** Significance was tested by Chi square $\left(\mathrm{X}^{2}\right)$

*Significance was tested by ANOVA.

${ }^{\$}$ Including non-CAUTI.

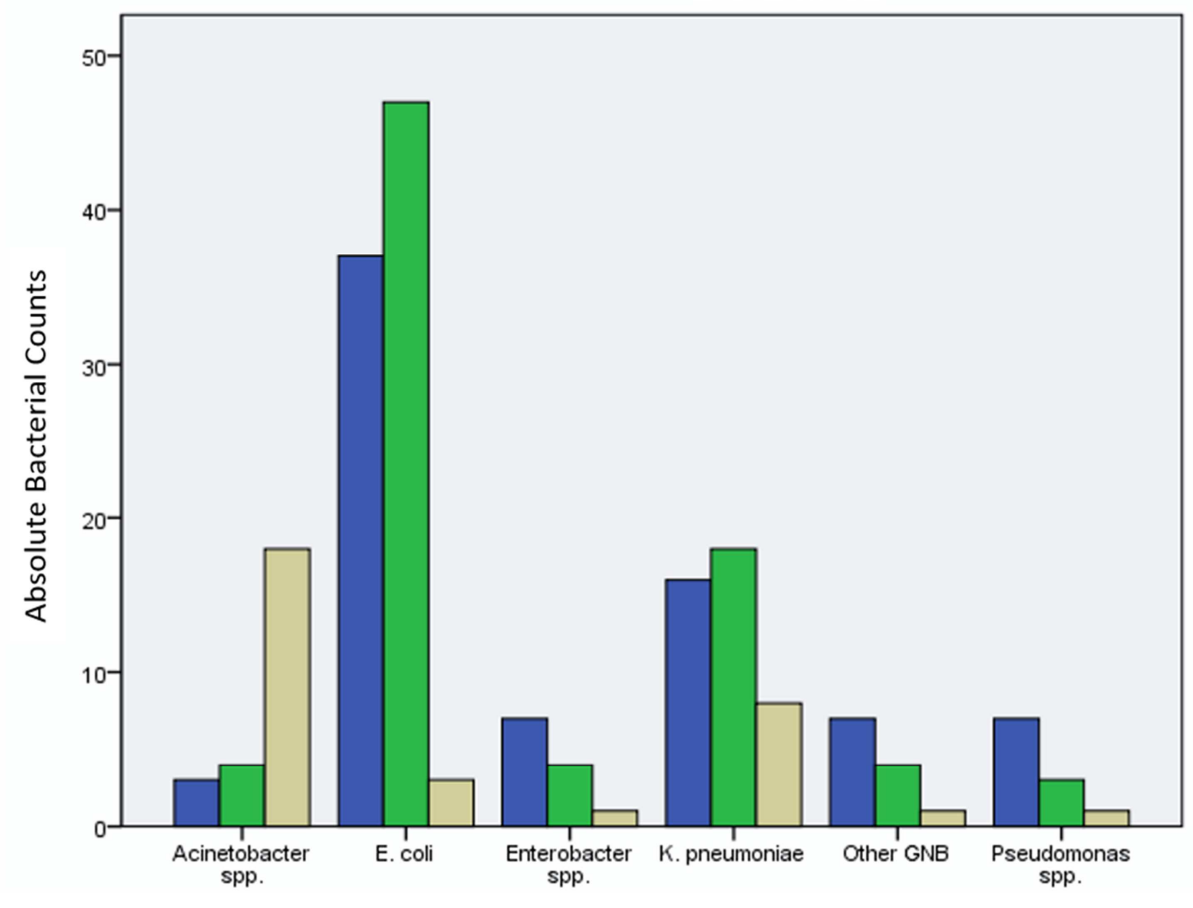

Other GNB: other gram-negative bacteria. Pseudomonas spp: including P. aeruginosa

Figure 1. The distribution of gram-negative bacteria cultured from patients with bloodstream infection, and their distribution according to the resistance patterns.

The outcomes; in-hospital and 28-days mortalities were significantly higher in the XDRGNB-BSI patients $(\mathrm{P}=$ 0.000 ), even after adjusting for appropriateness of the antimicrobial therapy mortalities were higher with XDRGNB-BSI patients for both endpoints $(\mathrm{P}=0.005$ and $\mathrm{P}$ $=0.003$ respectively). The length of the ICU stay was longer for XDRGNB-BSI patients than patients with the other two resistance patterns $(\mathrm{P}=0.000)$, but the length of hospital stay was not different for the three resistance patterns $(\mathrm{P}=0.413)$. Delay in antimicrobial therapy resulted in an increase in the length of hospital stay $(\mathrm{P}=0.025)$ and ICU stay $(\mathrm{P}=0.001)$ for only DSGNB-BSI patients, but not for patients with MDRGNB-BSI and XDRGNB-BSI ( $\mathrm{P} \geq 0.422$ ), (Table 2). Logistic regression analysis demonstrated no statistically significant interaction neither with the admission diagnoses $\left(P>0.3\right.$, Nagelkerke $\left.R^{2}=0.169\right)$ the antimicrobial class used $\left(\mathrm{P}>0.170\right.$, Nagelkerke $\mathrm{R}^{2}=0.444$, except for Aminoglycosides $\mathrm{P}=0.02)$, and the comorbidities $(\mathrm{P}>$ 0.263 . Nagelkerke $R^{2}=0.165$, except for solid tumor $P=$ $0.026)$ with the 28 days mortality. 
Table 2. The Outcome measures for patients with GNB-BSI distributed according to the antimicrobial susceptibility patterns.

\begin{tabular}{|c|c|c|c|c|}
\hline Characteristic & $\begin{array}{l}\text { DSGN-BSI } \\
\mathbf{N}=77\end{array}$ & $\begin{array}{l}\text { MDRGN-BSI } \\
\mathbf{N}=\mathbf{8 0}\end{array}$ & $\begin{array}{l}\text { XDRGN-BSI } \\
\mathbf{N}=32\end{array}$ & P-value ${ }^{\&}$ \\
\hline \multicolumn{5}{|l|}{ In-Hospital mortality } \\
\hline All patients & 9 & 20 & $20 *$ & $0.000 *$ \\
\hline \multicolumn{5}{|l|}{ Mortality 28 days } \\
\hline All patients & 16 & 23 & $25 * *$ & $0.000 * *$ \\
\hline Patients received appropriate therapy & 16 & 16 & 4 & 0.003 \\
\hline Length of Hospital stay & 12.79 & 11.67 & 16.72 & 0.413 \\
\hline Patients improved & 66 & 56 & 7 & $0.000^{\#}$ \\
\hline
\end{tabular}

BSI: blood stream infection. DSGNB: drug susceptible gram-negative bacteria. MDR: multidrug resistant. XDR: extensive drug resistant

${ }^{\&}$ Analyzed by one-way ANOVA for proportions.

*Tukey HSD shows significant difference between XDRGNB versus DSGNB $(\mathrm{P}=0.000)$, and XDRGNB versus MDRGNB $(\mathrm{P}=0.000)$, but not significance for MDRGNB versus DSGNB $(\mathrm{P}=0.101)$.

**Tukey HSD shows significant difference between XDRGNB versus DSGNB $(\mathrm{P}=0.000)$, and XDRGNB versus MDRGNB $(\mathrm{P}=0.000)$, but not significance for MDRGNB versus DSGNB ( $\mathrm{P}=0.479)$.

${ }^{\$}$ Tukey HDS showed that the difference was between XDRGNB versus DSGNB $(\mathrm{P}=0.000)$ and XDRGNB versus MDRGNB $(\mathrm{P}=0.000)$

\#Significantly less patients improved in XDRGNB-BSI arm when compared with the other two arms, also with MDRGNB-BSI arm though it was borderline when compared with DSBGNB-BSI $(\mathrm{P}=0.047)$.

The frequency of the inappropriate use of antimicrobials therapy was significantly more in patients with XDRGNBBSI than patients in the other two resistance patterns $(\mathrm{P}=$ 0.000), and improper indications like using aminoglycosides and quinolones as monotherapy $(\mathrm{P}=0.001)$, but not between DSGNB-BSI and MDRGNB-BSI patients $(\mathrm{P}=0.931)$. There was a significant difference in the delay of the antimicrobial therapy in patients with XDRGNB-BSI compared with DSGNB-BSI patients $(P=0.031)$ but not versus MDRGNB-
BSI $(\mathrm{P}=0.392)$. There were five CREs all in the XDRGNBBSI category. ESBL-producing bacteria were $63.1 \%$ in patients with the MDRGNB-BSI, 29.8\% in the DSGNB-BSI and $7.1 \%$ in the XDRGNB-BSI patients. In this group neither ESBL or CRE showed a significant statistical difference within the DSGNB, MDRGNB, and XDRGNB resistance patterns when were selected to test for in-hospital death, 28days death, length of ICU stay, hospital stay, appropriateness of therapy and improvement $(\mathrm{P}>0.05)$ (Table 3$)$.

Table 3. Appropriateness of antimicrobial therapy classified according to drug susceptibility patterns for patients with GNB-BSI.

\begin{tabular}{lllll}
\hline Antimicrobial Therapy & DSGNB-BSI & MDRGN-BSI & XDRGN-BSI \\
\hline Appropriate & 71 & 63 & 4 & P-Value \\
Used in a resistant bacterium $^{(}$ & 3 & 8 & 25 & 0.000 \\
Delayed $^{\#}$ & 4 & 11 & 0.000 & 0.032 \\
Improper in indication* $^{*}$ & 1 & 2 & 6 & 0.001 \\
\hline
\end{tabular}

BSI: blood stream infection. DSGNB: drug susceptible gram-negative bacteria. MDRGNB: multidrug resistant gram-negative bacteria. XDRGNB: extensive drug resistant gram-negative bacteria.

${ }^{\circledR}$ Significantly, resistant bacteria were more in the XDRGNB patients (included all CRE).

\# Not starting the appropriate antimicrobial until culture results were available $(\mathrm{P}=0.032)$, Tukey post hoc analysis revealed that the only significant difference is between XDRGNB-BSI patients and DSGNB-BSI patients (0.031) and not XDRGNB-BSI versus MDRGNB-BSI patients (P =0.392).

*Improper: using agents that are not labelled for blood stream infection as monotherapy like aminoglycosides and quinolones. The significant statistical difference was between XDRGNB-BSI and DSGNB-BSI patients, and XDRGNB-BSI and MDRGNB-BSI patients.

\section{Discussion}

Despite several studies that address mortality and length of hospital stay contrasted with inappropriate therapy $[12,13]$, few studies either focused on monomicrobial resistance like Acinetobacter and Pseudomonas as a cause of inappropriate empiric therapy and mortality, while another study invited to look at resistance as a major part of inappropriate therapy $[14,15,9]$. Our study directly asses resistance patterns according to an international experts proposal classification for the definition of XDR and MDR gram-negative bacteria (excluding the pan drug-resistant bacteria PDRGNB for being not present in our patients) and their impact on mortality and length of ICU and hospital stay [3, 7] In the quest to analyze our data based on the unique resistance patterns in relation to the outcomes, we tried our best to adjust for several confounders and lurking variables like age, gender, SOFA and APACHE II scores, initial admission diagnoses, comorbidities, the infecting bacterial species, other classified resistance patterns like ESBL, CRE, and types of bacteria based on lactose fermentation. We found that all were statistically not different among the three groups $(P>0.05)$ except significantly few more cases in VAP were in the XDRGNB-BSI patients, nonetheless their absolute numbers were modest, this was to some extent reassuring that the bulk of the studied outcomes were dominantly correlated with the three resistance patterns under study i.e XDRGNB, MDRGNB and DSGNB.

Despite the XDRGNB-BSI patients did have more inappropriate initial empiric coverage due to resistant 
bacteria, but the inappropriate therapy may affect mortality independent of the resistance pattern [16, 17]. In our patients, those with XDRGNB-BSI infection carried significantly higher mortality compared with DSGNB-BSI and MDRGNB-BSI patients $(\mathrm{P}=0.000)$, even after adjusting for patients receiving appropriate therapy for their resistant bacteria the hospital mortality was higher $(\mathrm{P}=0.005)$ and the 28 -days mortality $(\mathrm{P}=0.003)$. Those who were discharged home, the clinical improvement was significantly less in patients with XDRGNB-BSI compared with patients in the other two resistance patterns $(\mathrm{P}=0.000)$.

Like what was found in other studies, resistance is a cause for a longer hospital stay. Here, XDRGNB-BSI subset of patients have longer ICU length of stay $(\mathrm{P}=0.000)$ but not the overall hospital stay $(\mathrm{P}=0.413)$, this may be due to the nature of the most uninsured patients in the private costly hospitals, patients and families tend to discharge their patients as soon as they become reasonably stable [18]. Other studies did not show that resistance is a cause for longer hospital length of stay, but their definition of resistance in their patients was based on ceftazidime resistance in gramnegative bacteria [19]. The delay in the antimicrobial therapy resulted in the increase in the length of hospital stay $(\mathrm{P}=$ $0.025)$ and ICU stay $(P=0.001)$ for only DSGNB-BSI patients, but not for patients with MDRGNB-BSI and XDRGNB-BSI ( $\mathrm{P} \geq 0.422$ ), possibly due to a concealed effect of the longer stay for patients with both resistance patterns, reflecting their comorbid status [20]. Newly introduced automated testing systems such as MALDI-TOF MS proved useful in reducing mortality and hospital length of saty, especially in patients with gram-negative sepsis [21].

\section{Conclusion}

Patients with BSI due to XDRGNB have higher mortality and ICU length of stay. Delayed antimicrobial therapy caused an increase in the length of hospital stay and ICU stay in patients with DSGNB, but not XDRGNB and MDRGNB, possibly due to their comorbid conditions. Multiclass antimicrobials resistance adds more outcome-adverse effect on patients, and this phenomenon is not unique for ESBLproducing gram-negative bacteria and CRE as usually reported.

\section{References}

[1] Helen W. Boucher, George H. Talbot, Daniel K. Benjamin Jr, John Bradley, Robert J. Guidos, Ronald N. Jones, Barbara E. Murray, Robert A. Bonomo, and David Gilbert, for the Infectious Diseases Society of Americaa10 × '20 ProgressDevelopment of New Drugs Active Against Gram-Negative Bacilli: An Update From the Infectious Diseases Society of America. Clinical Infectious Diseases 2013; 56 (12): 1685-94.

[2] McGowan Jr JE. Resistance in nonfermenting gram-negative bacteria: multidrug resistance to the maximum. American journal of infection control. 2006 Jun 1; 34 (5): S29-37.

[3] Souli M, Galani I, Giamarellou H. Emergence of extensively drug-resistant and pan-drug-resistant Gram-negative bacilli in Europe. Eurosurveillance. 2008 Nov 20; 13 (47): 19045.

[4] Stijn Blot, Koenraad Vandewoude, Dirk De Bacquer, and Francis Colardyn. Nosocomial Bacteremia Caused by Antibiotic-Resistant Gram-Negative Bacteria in Critically Ill Patients: Clinical Outcome and Length of Hospitalization. Clinical Infectious Diseases 2002; 34: 1600-6.

[5] Yu-Chen Tseng, Jann-Tay Wang, Fe-Lin Lin Wu, Yee-Chun Chen, Wei-Chu Chie, Shan-Chwen Chang. Prognosis of adult patients with bacteremia caused by extensively resistant Acinetobacter baumannii. Diagnostic Microbiology and Infectious Disease (2007); 59: 181-190.

[6] Bodro, Marta, Sabé, Núria, Tubau, Fe, Lladó, Laura, Baliellas, Carme, Roca, Josep Cruzado, Josep, Maria, Carratalà, Jordi. Risk Factors and Outcomes of Bacteremia Caused by DrugResistant ESKAPE Pathogens in Solid-Organ Transplant Recipients. Transplantation: 15 November 2013 - Volume 96 Issue 9 - p 843-849. doi: 10.1097/TP.0b013e3182a049fd.

[7] Magiorakos AP, Srinivasan A, Carey RB, Carmeli Y, Falagas ME, Giske CG, Harbarth S, Hindler JF, Kahlmeter G, OlssonLiljequist B, Paterson DL, Rice LB, Stelling J, Struelens MJ, Vatopoulos A, Weber JT and Monnet DL. Multidrug-resistant, extensively drug-resistant and pan-drug-resistant bacteria: an international expert proposal for interim standard definitions for acquired resistance. Clin Microbiol Infect 2012; 18: 268281. 10.1111/j.1469-0691.2011.03570.x.

[8] Luna CM, Vujacich P, Niederman MS et al. Impact of BAL data on the therapy and outcome of ventilator-associated pneumonia. Chest 1997; 111: 676-685.

[9] Davey P G and Marwick C. Appropriate vs. inappropriate antimicrobial therapy. Clin Microbiol Infect 2008; 14 (Suppl. 3): $15-21$.

[10] Ibrahim E H, Sherman G, Ward S, Fraser V J and Kollef M H. The Influence of Inadequate Antimicrobial Treatment of Bloodstream Infections on Patient Outcomes in the ICU Setting. Chest 2000; 118; 146-155. DOI: 10.1378/chest.118.1.146.

[11] R. P. Dellinger, Mitchell M. Levy, Andrew Rhodes, Djillali Annane, Herwig Gerlach, Steven M. Opal, Jonathan E. Sevransky, Charles L. Sprung, Ivor S. Douglas, Roman Jaeschke, Tiffany M. Osborn, Mark E. Nunnally, Sean R. Townsend, Konrad Reinhart, Ruth M. Kleinpell, Derek C. Angus, Clifford S. Deutschman, Flavia R. Machado, Gordon D. Rubenfeld, Steven Webb, Richard J. Beale, Jean-Louis Vincent, Rui Moreno. The Surviving Sepsis Campaign Guidelines Committee including The Pediatric Subgroup* Surviving Sepsis Campaign: International Guidelines for Management of Severe Sepsis and Septic Shock, 2012. Intensive Care Med (2013) 39: 165-228. DOI $10.1007 / \mathrm{s} 00134-012-2769-8$.

[12] Cain SE, Kohn J, Bookstaver PB, Albrecht H, Al-Hasan MN. Stratification of the impact of inappropriate empirical antimicrobial therapy for Gram-negative bloodstream infections by predicted prognosis. Antimicrobial agents and chemotherapy. 2015 Jan 1; 59 (1): 245-50.

[13] Battle SE, Bookstaver PB, Justo JA, Kohn J, Albrecht H, AlHasan MN. Association between inappropriate empirical antimicrobial therapy and hospital length of stay in gram-negative bloodstream infections: stratification by prognosis. Journal of Antimicrobial Chemotherapy. 2016 Sep 28; 72 (1): 299-304. 
[14] Zilberberg MD, Nathanson BH, Sulham K, Fan W, Shorr AF. Multidrug resistance, inappropriate empiric therapy, and hospital mortality in Acinetobacter baumannii pneumonia and sepsis. Critical Care. 2016 Dec; 20 (1): 221.

[15] Merchant S, Proudfoot EM, Quadri HN, McElroy HJ, Wright WR, Gupta A, Sarpong EM. Risk factors for Pseudomonas aeruginosa infections in Asia-Pacific and the consequences of inappropriate initial antimicrobial therapy: A systematic literature review and meta-analysis. Journal of global antimicrobial resistance. 2018 Sep 1; 14: 33-44.

[16] Harbarth S, Garbino J, Pugin J, Romand JA, Lew D, Pittet D. Inappropriate initial antimicrobial therapy and its effect on survival in a clinical trial of immunomodulating therapy for severe sepsis. The American journal of medicine. 2003 Nov 1; 115 (7): 529-35.

[17] Cheong HS, Kang CI, Wi YM, Ko KS, Chung DR, Lee NY, Song JH, Peck KR. Inappropriate initial antimicrobial therapy as a risk factor for mortality in patients with community-onset Pseudomonas aeruginosa bacteremia. European journal of clinical microbiology \& infectious diseases. 2008 Dec 1; 27 (12): 1219-25.
[18] Mauldin PD, Salgado CD, Hansen IS, Durup DT, Bosso JA. Attributable hospital costs and length of stay associated with healthcare-associated infections caused by antibiotic-resistant gram-negative bacteria. Antimicrobial agents and chemotherapy. 2010 Jan 1; 54 (1): 109-15.

[19] Blot S, Vandewoude K, De Bacquer D, Colardyn F. Nosocomial bacteremia caused by antibiotic-resistant gramnegative bacteria in critically ill patients: clinical outcome and length of hospitalization. Clinical infectious diseases. 2002 Jun 15; 34 (12): 1600-6.

[20] Zhang D, Micek ST, Kollef MH. Time to appropriate antibiotic therapy is an independent determinant of postinfection ICU and hospital lengths of stay in patients with sepsis. Critical care medicine. 2015 Oct 1; 43 (10): 2133-40.

[21] Beganovic M, Costello M, Wieczorkiewicz SM. Effect of matrix-assisted laser desorption ionization-time of flight mass spectrometry (MALDI-TOF MS) alone versus MALDI-TOF MS combined with real-time antimicrobial stewardship interventions on time to optimal antimicrobial therapy in patients with positive blood cultures. Journal of clinical microbiology. 2017 May 1; 55 (5): 1437-45. 